\title{
Ripple-triggered stimulation of the locus coeruleus during post-learning sleep disrupts ripple/spindle coupling and impairs memory consolidation
}

\author{
Yulia Novitskaya, ${ }^{1}$ Susan J. Sara, ${ }^{2,3}$ Nikos K. Logothetis, ${ }^{1,4}$ and Oxana Eschenko ${ }^{1}$ \\ ${ }^{1}$ Max Planck Institute for Biological Cybernetics, Tubingen 72076, Germany; ${ }^{2}$ Center for Integrative Research in Biology, CNRS- \\ UMR7152, Collège de France, Paris 75005, France; ${ }^{3}$ Department of Child and Adolescent Psychiatry, New York University Medical \\ School, New York, New York 10016, USA; ${ }^{4}$ Centre for Imaging Sciences, Biomedical Imaging Institute, University of Manchester, \\ Manchester M13 9PT, United Kingdom
}

\begin{abstract}
Experience-induced replay of neuronal ensembles occurs during hippocampal high-frequency oscillations, or ripples. Postlearning increase in ripple rate is predictive of memory recall, while ripple disruption impairs learning. Ripples may thus present a fundamental component of a neurophysiological mechanism of memory consolidation. In addition to systemlevel local and cross-regional interactions, a consolidation mechanism involves stabilization of memory representations at the synaptic level. Synaptic plasticity within experience-activated neuronal networks is facilitated by noradrenaline release from the axon terminals of the locus coeruleus (LC). Here, to better understand interactions between the system and synaptic mechanisms underlying "off-line" consolidation, we examined the effects of ripple-associated LC activation on hippocampal and cortical activity and on spatial memory. Rats were trained on a radial maze; after each daily learning session neural activity was monitored for $1 \mathrm{~h}$ via implanted electrode arrays. Immediately following "on-line" detection of ripple, a brief train of electrical pulses $(0.05 \mathrm{~mA})$ was applied to LC. Low-frequency $(20 \mathrm{~Hz})$ stimulation had no effect on spatial learning, while higher-frequency $(100 \mathrm{~Hz})$ trains transiently blocked generation of ripple-associated cortical spindles and caused a reference memory deficit. Suppression of synchronous ripple/spindle events appears to interfere with hippocampal-cortical communication, thereby reducing the efficiency of "off-line" memory consolidation.
\end{abstract}

[Supplemental material is available for this article.]

Early reports of experience-induced replay of neuronal ensembles (Pavlides and Winson 1989; Wilson and McNaughton 1994; Skaggs and McNaughton 1996) became the first empirical evidence for Marr's postulation of spontaneous reactivation of memory traces (Marr 1971). Since these seminal discoveries, we have seen an avalanche of studies exploring neurophysiological mechanisms underlying so-called systems-level memory consolidation, a process that enables newly encoded memory representations (presumably in hippocampus) to be transferred and redistributed within a large-scale neuronal networks for long-term storage. Current experimental evidence and theory support the fundamental role of hippocampal-cortical interactions occurring "offline", i.e., when the brain is not processing external input, for memory consolidation (Buzsaki 1989; Pennartz et al. 2002; Diekelmann and Born 2010; Battaglia et al. 2011).

The sharp wave ripple complexes (SPW-Rs) are observed in hippocampus during off-line behavioral states and are thought to present a fundamental component of a neurophysiological mechanism of memory consolidation. Specifically, the SPW-Rs mark time windows of increased cellular excitability (Buzsaki 1985), they are indicative of replay of experience-activated neuronal sequences (Kudrimoti et al. 1999; Nadasdy et al. 1999) and their occurrence during slow wave sleep (SWS) is temporally locked to periods of enhanced cortical excitability, or up-states (Siapas and Wilson 1998; Molle et al. 2006). Thus, SPW-Rs may enable temporally precise coordinated discharge of recently activat-

Corresponding author: oxana.eschenko@tuebingen.mpg.de Article is online at http://www.learnmem.org/cgi/doi/10.1101//m.040923.115. ed neurons and serve as an intrinsic high-frequency source triggering synaptic plasticity within an extended neural network representing new memories (Buzsaki 1989). Moreover, ripple activity is increased after learning and post-learning ripple rate is predictive of memory recall (Eschenko et al. 2008; Ramadan et al. 2009). Sustained increase in ripple activity after learning is thought to reflect experience-induced changes in cellular excitability and synaptic connectivity within recently activated hippocampal networks (King et al. 1999). This view is further supported by experimental evidence that learning induces persistent changes in synaptic transmission within experience-activated neural networks (Saar and Barkai 2003; Zhang and Linden 2003).

Many experimental findings and theories suggest that Hebbian plasticity alone is not sufficient for producing longlasting synaptic changes and that accompanying neuromodulatory mechanisms are critical for maintaining synaptic modifications, which eventually lead to enduring memories (Bailey et al. 2000). It is well-established that the noradrenaline (NE) release facilitates synaptic plasticity in hippocampus by increasing the magnitude, duration, and probability of LTP induction (Harley and Sara 1992; Gelinas and Nguyen 2005). Furthermore, there is a large body of literature implicating the LC-NE system in early stages of memory consolidation, taking place during and

\footnotetext{
(C) 2016 Novitskaya et al. This article is distributed exclusively by Cold Spring Harbor Laboratory Press for the first 12 months after the full-issue publication date (see http://learnmem.cshlp.org/site/misc/terms.xhtml). After 12 months, it is available under a Creative Commons License (AttributionNonCommercial 4.0 International), as described at http://creativecommons. org/licenses/by-nc/4.0/.
} 
immediately after new experience (Sara 2000; Kobayashi and Yasoshima 2001; McGaugh 2013). Much less is known about the role of LC-NE system for off-line consolidation, although earlier pharmacological studies suggested a critical involvement of $\mathrm{NE}$ modulation at a later phase ( $\sim 2 \mathrm{~h}$ post-learning) of memory consolidation (Sara et al. 1999; Tronel et al. 2004). Earlier we reported that LC neurons, usually quiescent during sleep, show intermittent bursting activity during SWS following a learning experience. Notably, increased LC firing during post-learning SWS was not observed in poor learners (Eschenko and Sara 2008). Subsequent study revealed that LC discharge precedes the cortical up-state (Eschenko et al. 2012).

After years of discordant results, mostly from in vitro studies, it has recently been confirmed, in vivo, that strict temporal association between LC activation and the perforant path input to the dentate gyrus of the hippocampus is necessary to induce both long-lasting changes in cellular excitability, typically seen in the presence of NE, and long-lasting synaptic facilitation, indicated by changes in the slope of the EPSP (Reid and Harley 2010). Since SPW-Rs and associated replay are temporally aligned to cortical up-states and sleep spindles (Siapas and Wilson 1998; Battaglia et al. 2004; Molle et al. 2006), we reasoned that LC phasic activation and associated NE release precisely at times of hippocampal-cortical interaction would provide ideal conditions for NE-facilitated synaptic plasticity within recently activated neural networks and therefore result in improved memory performance. We tested this hypothesis by training rats on a spatial discrimination task and applying brief trains of electric pulses to LC after learning at times of SPW-Rs occurrence.

\section{Results}

\section{Effects of post-learning phasic LC activation on spatial task acquisition}

Rats were trained on a radial maze task over $8 \mathrm{~d}$; each daily training session consisted of three trials and the trial ended when a rat either collected rewards available at the end of three fixed maze arms or after 5 min elapsed. During the $1 \mathrm{~h}$ rest period after each training session brief trains of electric pulses $(0.05 \mathrm{~mA})$ were delivered to the LC unilaterally at times of ripple occurrence (ripple-triggered, RT) or at random. In the case of the rippletriggered condition, we tested the effects of low- and highfrequency LC stimulation (RT-LFS and RT-HFS, respectively). The LFS approximated spontaneous burst firing of the LC-NE neurons (Aston-Jones and Bloom 1981) and consisted of five pulses applied at $20 \mathrm{~Hz}$ after every detected ripple. According to earlier studies, HFS (50-100 Hz) elicits robust burst of firing of LC-NE neurons, produces sustained firing of prefrontal neurons (Marzo et al. 2014), and induces substantial NE release from the LC terminals (Florin-Lechner et al. 1996). Special care was taken that neither stimulation parameter elicited any observable behavioral response or awakening of the sleeping rat (Supplemental Fig. 1; also see Materials and Methods for details). Figure 1 illustrates the timing of LC stimulation at ripple onset detected "on-line". The total number of ripple-triggered stimulations depended on spontaneous behavior, as no stimulation was applied during the active awake state when hippocampal ripples rarely occur. In the case of random stimulation, HFS was used and the timing of current application approximated the ripple-triggered condition. Importantly, the number of stimulations was stable across days (repeated-measures ANOVA, day effect: $F_{(6,13)}=1.89$, ns); there was neither a day $\times$ group interaction $\left(F_{(12,28)}=1.37\right.$, ns) nor a significant group effect $\left(F_{(2,18)}=0.43, \mathrm{~ns}\right)$. Overall, rats assigned to different stimulation conditions (RT-LFS, RT-HFS, or randomHFS) received a comparable total number of stimulations per session (418.4 \pm 38.6 ; $\min / \max : 83 / 830)$. The RT-LFS did not affect the SWS-associated power spectrum of the cortical EEG averaged over all SWS episodes of 1-h recording session (Fig. 2A; Supplemental Fig. 2); there was also no post-stimulus spectral change at any frequency range (Fig. 2B, left panel). In contrast, HFS produced a transient ( $\sim 1 \mathrm{sec})$ gamma $(30-90 \mathrm{~Hz})$ power increase in cortical field potentials accompanied by strong decreases in the low $(<30 \mathrm{~Hz})$ frequency EEG component (Fig. 2B, right panel; Supplemental Fig. 2). This transient change of cortical activity elicited by each HFS-train did not, however, affect the overall SWS-associated EEG power spectrum (Fig. 2A).

We first examined the effects of RT-LFS on spatial learning by comparing the accuracy of task performance between LC-stimulated and intact rats. Preliminary analysis revealed that there

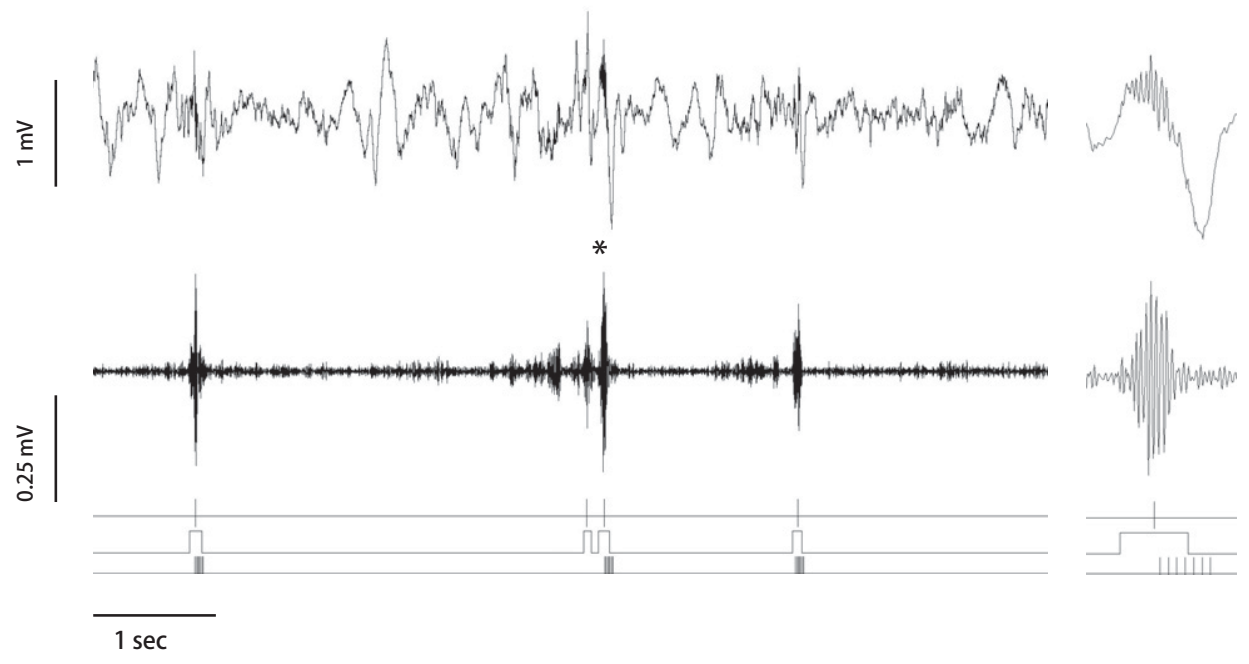

Figure 1. Ripple-triggered phasic LC stimulation. Extract of the raw (top trace) and band-pass $(120-240 \mathrm{~Hz})$ filtered CA1-LFP signal. The ripple peaks, on/off sets, and pulse trains (10 pulses, $0.05 \mathrm{~mA}$ at $100 \mathrm{~Hz}$ ) delivered to the $\mathrm{LC}$ are shown on three bottom traces. Asterisk indicates the ripple that is illustrated at higher temporal resolution on the right panel. Note LC stimulation was applied immediately after ripple onset and did not affect generation of the ongoing ripple. 
A

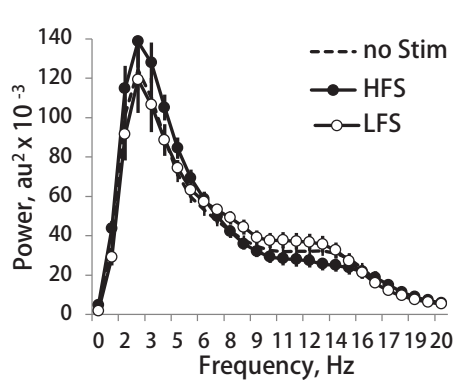

B

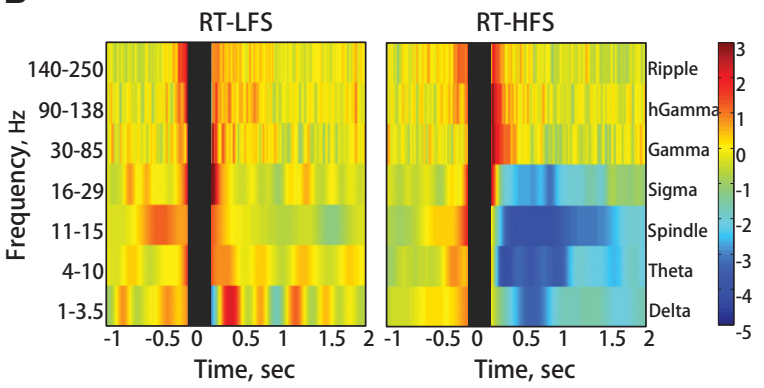

Figure 2. Effects of phasic LC stimulation on cortical EEG. (A) EEG power spectrum averaged over all SWS episodes during 1-h recording session. Dashed line: control recording without LC stimulation, solid lines with open and filled circles: LFS- and HFS-groups, respectively. ( $B$ ) The group averages of prestimulus normalized band-limited power of cortical EEG around the stimulation onset are shown for RT-LFS (left panel) and RT-HFS (right panel). The BLP for each frequency range was normalized to $2 \mathrm{sec}$ prestimulus interval and converted to $z$-scores. Gray bar indicates duration of the pulse train, a color bar represents $z$-scores. Note a transient frequency-specific power modulation elicited by the HFS, and the absence of the EEG spectral change in the case of the LFS.

was no difference in learning rate between intact and shamoperated rats (data not shown). To this end, for each trial we counted the proportion of entries to the rewarded maze arms (correct choices) out of the total number of maze arms visited and submitted the session averages over $8 \mathrm{~d}$ of training for statistical analysis. The performance accuracy improved equally over time in intact and LC-stimulated rats (Fig. 3A); this observation was confirmed by the repeated-measures analysis of variance (ANOVA). There was a significant effect of repetition $\left(F_{(7,11)}=\right.$ 15.25, $P<0.001$, multivariate Pillai's Trace test), but neither significant day $\times$ group interaction $\left(F_{(7,11)}=0.47, \mathrm{~ns}\right)$, nor overall group effect $\left(F_{(1,17)}=1.10, \mathrm{~ns}\right)$. Likewise, the trial time gradually decreased (Fig. 3B). The repeated-measures ANOVA revealed a strong day effect $\left(F_{(7,11)}=82.66, P<0.001\right)$, but neither day $\times$ group interaction $\left(F_{(7,11)}=0.51\right.$, ns), nor between-group difference $\left(F_{(1,17)}=1.57, \mathrm{~ns}\right)$. Finally, intact and LC-stimulated rats had a similar number of reference (entries to unbaited maze arms) or working (reentries to visited maze arms) memory errors ( $P>0.05$ for between-group comparisons). Thus, the low-frequency stimulation trains applied to LC around times of ripple occurrence for $1 \mathrm{~h}$ after each learning session did not affect the acquisition rate of a spatial discrimination task.

Two additional groups received high-frequency LC stimulation either during ripples or randomly for $1 \mathrm{~h}$ after each training session, and their maze performance was compared with intact controls. The performance accuracy improved over time in all three experimental groups, however at a much slower rate in the RT-HFS group (Fig. 3C). The repeated-measures ANOVA showed no significant day $\times$ group interaction $\left(F_{(14,38)}=0.73, \mathrm{~ns}\right)$, but a highly significant overall group effect $\left(F_{(2,24)}=7.62\right.$, $P=0.003)$ confirming that learning was impaired in the RT-HFS group. Figure 3D shows that trial duration in the RT-HFS group was not decreasing over training, as in the other two groups (day $\times$ group: $F_{(14.38)}=2.06, \quad P<0.05$; group: $\left.F_{(2,24)}=7.44, P<0.01\right)$. In order to elucidate the nature of the learning deficit, we analyzed behavioral variables in more detail. The learning efficiency in this maze task depends on two factors: (1) the number of collected rewards and (2) the total number of maze arms visited. Comparison of these two factors among the three experimental groups showed that the learning deficit originated from the fact that rats from the RT-HFS group consistently retrieved fewer than the maximum (3) available rewards (day effect: $F_{(7,18)}=2.80, P<0.05$; day $\times$ group interaction: $F_{(14.38)}=0.54$ ns; group effect: $\left.F_{(2,24)}=4.81, P<0.05\right)$. On the last day of training, rats from the RT-HFS group collected on average $2.32 \pm 0.26$ rewards per trial $(\min / \max : 1 / 3)$, while all other rats regularly retrieved all three rewards in each trial (Fig. 4A). The total number of maze arms visited and the number of reference or working memory errors did not differ among experimental conditions (all $P$-values $>0.05$ for between-group comparisons). Thus, a longer trial time in combination with incomplete reward collection
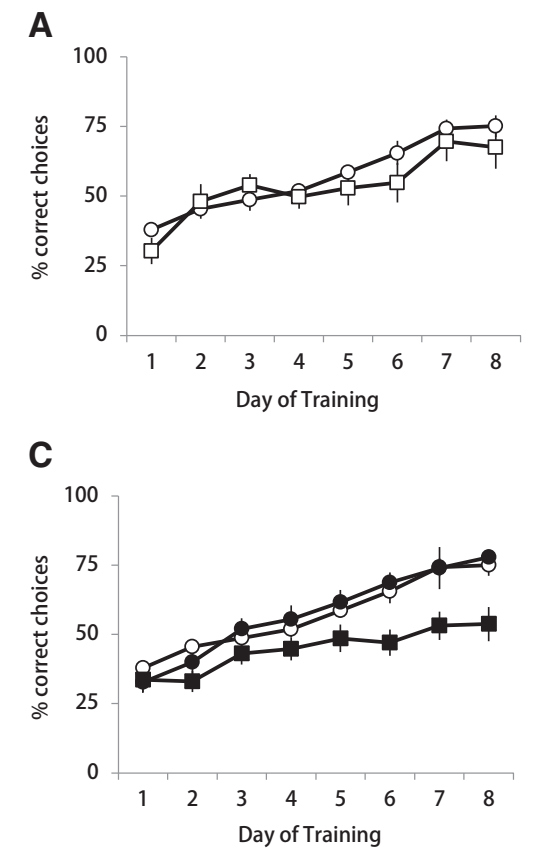

B

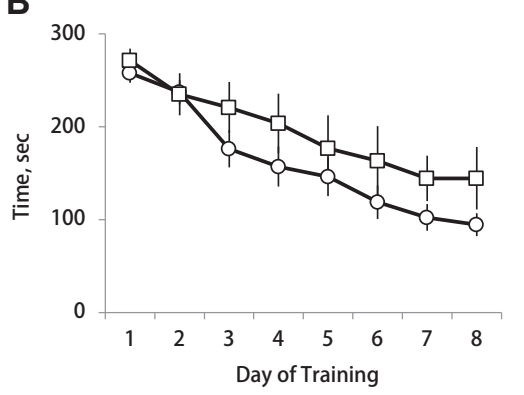

D

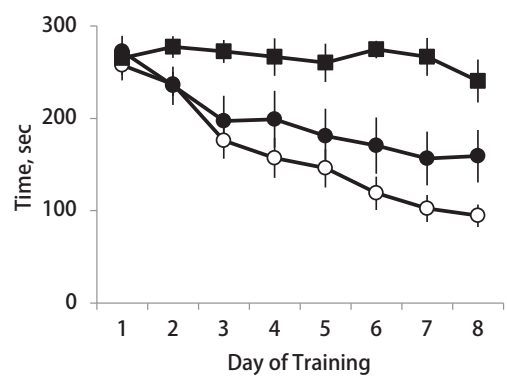

Figure 3. Effects of LC stimulation on a spatial task acquisition. Average performance accuracy $(A, C)$ and trial time $(B, D)$ are plotted across eight daily training sessions for different experimental groups. (Open circles) nonstimulated control, (open squares) RT-LFS, (filled squares) RT-HFS, and (filled circles) random-HFS. Note strong learning deficit in the RT-HFS group. 
A

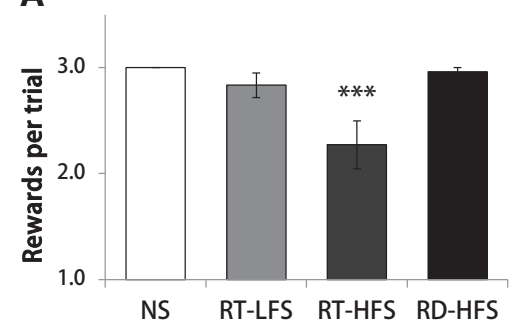

B

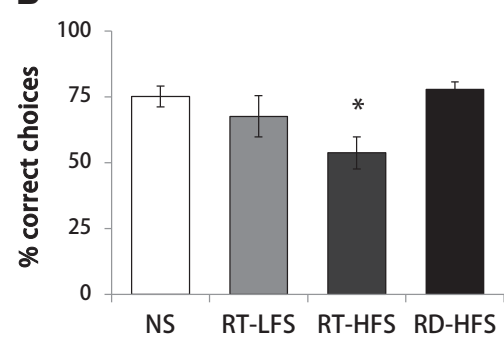

C

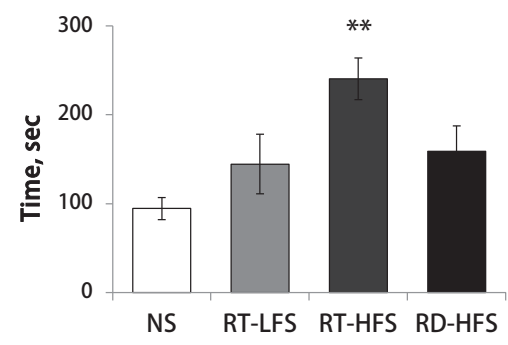

Figure 4. Post-learning ripple-triggered $L C$ activation causes a reference memory deficit. Bars represent the group means on the last day of training for the number of rewards retrieved $(A)$, performance accuracy $(B)$, and trial time (C). Note significant learning deficit was present exclusively in the RT-HFS group. $\left({ }^{*}\right) P<0.05,\left({ }^{* *}\right) P<0.01$, and $\left({ }^{* * *}\right) P<0.001$ compared with nonstimulated control (Dunnett's test). (NS) nonstimulated control, (RT-LFS) ripple-triggered low-frequency LC stimulation, (RT-HFS) ripple-triggered high-frequency LC stimulation, (RD-HFS) random high-frequency LC stimulation.

was most likely due to prolonged immobile states (e.g., sitting, grooming) on the maze. Such behaviors were occasionally noted by the experimenter, but post hoc quantitative analysis was precluded because there was no video monitoring.

Finally, we compared performance accuracy, trial time, and number of retrieved rewards on the last day of training across all four experimental conditions. One-way ANOVA revealed a significant group effect for each of the variables analyzed (accuracy: $F_{(3,32)}=4.07, P<0.05$; trial time: $F_{(3,32)}=7.29, P=0.001$; rewards: $\left.F_{(3,32)}=10.16, P<0.001\right)$. Subsequent post hoc Dunnett tests showed that the RT-HFS group differed from intact animals in all three behavioral measures, while no other comparisons were significant (Fig. 4). Overall, this pattern of results indicated a reference memory deficit (i.e., remembering rewarded locations on the maze) in the rats that received high-frequency stimulation trains to LC during ripples.

\section{Effects of phasic LC activation on occurrence of sleep spindles and hippocampal ripples}

To understand the physiological mechanisms that could account for the learning deficit observed in the RT-HFS group, we examined in detail effects of LC stimulation on neural activity in cortex and hippocampus. Specifically, we calculated the probability of spindle and ripple events around stimulation times. In cases of the ripple-triggered stimulation (RT-LFS and RT-HFS), the probability of ripples was expectedly high around the stimulation onset (Fig. 5A). The HFS resulted in transient suppression of spindle and ripple occurrence regardless of the timing of stimulation (RT or random), while no such effect was present in case of RT-LFS (Figs. 5A,B; Supplemental Fig. 2). The ripple/spindle suppression lasted for at least 1-sec post-stimulus time (Figs. 5A,B). The repeated-measures ANOVA performed on eight 500-msec bins during \pm 2 -sec peri-stimulus time confirmed this observation, as there was a significant effect of bin (spindles: $F_{(7,126)}=$ $17.92, P<0.001$; ripples: $\left.F_{(7,111)}=36.03, P<0.001\right)$ and a significant bin $\times$ group interaction (spindles: $F_{(14,254)}=5.20, P<0.001$; ripples: $F\left({ }_{14,224)}=21.34, P<0.001\right)$. Subsequent post hoc tests (paired-t-tests for each 500-msec bin) showed that in cases of HFS the post-stimulation event probability was significantly below the prestimulation level for up to $1.5 \mathrm{sec}$ for ripples and up to $2 \mathrm{sec}$ for spindles. Moreover, in the RT-HFS group, the poststimulus ripple probability was substantially lower than one expected from the interripple interval estimated from the baseline (no stimulation) recordings (Fig. 5C). Finally, both HFS conditions (RT or random) resulted in a substantial decrease of sleep spindle probability within the most frequent interevent interval of 1 to $4 \mathrm{sec}$ (Fig. 5D). Thus, the HFS applied to LC at times of ripple occurrence prevented generation of the ripple-associated sleep spindles; the latter effect could potentially underlie the memory deficit observed exclusively under this experimental condition.

\section{Effects of LC stimulation on awake/sleep pattern and sleep-associated neural activity}

The frequency and timing of LC stimulation in relation to hippocampal ripples appeared to be the critical factors, with only HFS temporally linked to ripples producing memory deficit. To eliminate possible effects of LC stimulation on overall awake/ sleep pattern or sleep-associated neural activity in cortex and hippocampus, we analyzed electrophysiology data obtained during the baseline 1-h recording session (without LC stimulation or preceding learning experience) and the data obtained during postlearning recording sessions when LC stimulation was applied.

We first ruled out possible cumulative effects related to repeated application of LC stimulation over seven daily postlearning sessions by submitting a number of behavioral and physiological variables to the repeated-measures ANOVA. The statistical results showed that the total time spent awake or asleep was stable across days (all $P$-values $>0.05$ ). Despite substantial within-group variability, on average, rats spent an equal proportion of time $(\sim 30 \%)$ in active awake (e.g., exploration, locomotion, grooming), quiet awake (immobile awake), or spindle-rich SWS (Table 1). Other behavioral states, like spindle-free SWS and REM sleep were rare or essentially absent during the 1-h observation period, and therefore they were not further analyzed. Also, there was no significant effect of repetition for the occurrence of slow waves, sleep spindles, and ripples-the hallmark neural events characterizing SWS (all $P$-values $>0.05$ ). Based on these results, for further analyses the mean values over seven sessions with LC stimulation were compared with corresponding values during baseline recording when neither LC stimulation nor preceding learning experience took place. Table 1 summarizes the results for all variables analyzed and for all experimental conditions.

Repeated-measures ANOVA revealed a significant effect of repetition (baseline versus LC stimulation) for the total time spent in the quiet awake-state and spindle-rich SWS (quiet awake: $F_{(1,19)}=10.62, P<0.01$ and SWS: $\left.F_{(1,19)}=17.89, P<0.001\right)$. As shown in the Table 1 , in post-learning sessions with LC 
A

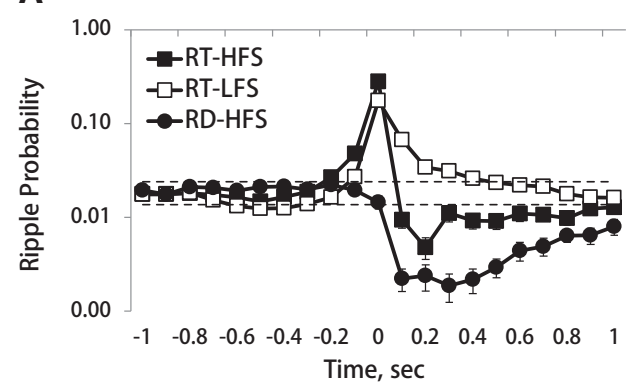

B

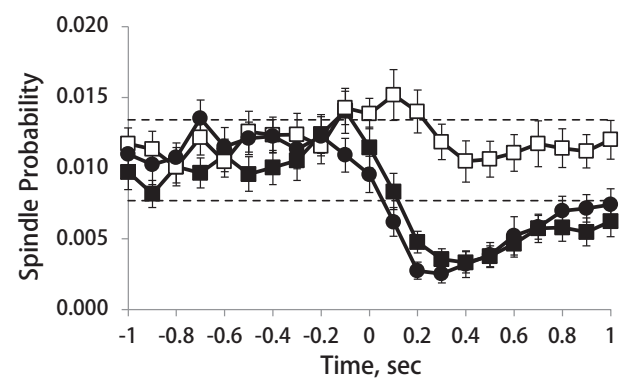

C

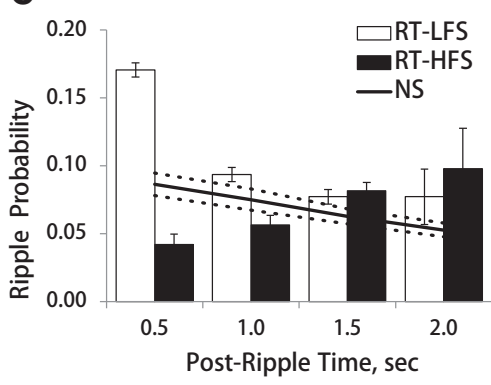

D

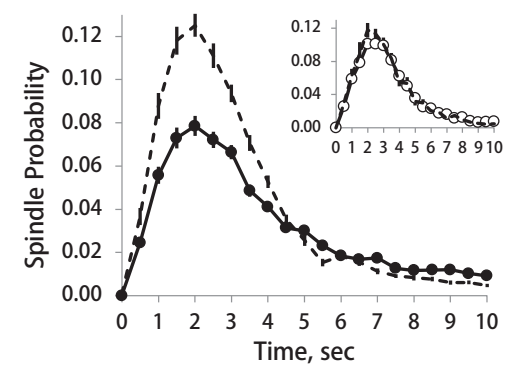

arousal level after maze exploration and the learning experience. More specific effects of LC stimulation on the SWS-associated neural activity in cortex, such as suppression of the high-amplitude delta waves in the case of ripple-triggered (LFS and HFS), or sleep spindles in randomly stimulated rats, are also not likely to cause the memory deficit observed only in the RT-HFS group. Thus, a transient elimination of simultaneously occurring ripple/spindle events caused by the high-frequency LC stimulation applied during ripples appears the most plausible factor underlying memory deficit in the RT-HFS group.

\section{Discussion}

The main result of the present study is that a brief ( $\sim 100-200 \mathrm{~ms})$ high-frequency $(\sim 100 \mathrm{~Hz})$ stimulation of LC during the post-learning period at times of SPW-Rs causes a spatial memory deficit. In addition to this behavioral effect, phasic LC activation induces a transient shift in the hippocampal-cortical network state that did not permit the occurrence of coupled spindle/ripple events. Phasic NE release by the LC terminals in forebrain targets including hippocampus and cortex decreased the level of synchronized neural activity and made ripple and spindle generation temporarily $(\sim 1 \mathrm{sec})$ impossible. Importantly, the timing of LC stimulation was critical for producing the memory impairment as and spindles $(B)$ around onset of LC stimulation $(t=0)$. (Open squares) RT-LFS, (filled squares) RT-HFS, and (filled circles) random-HFS. Dashed lines indicate lower and upper $95 \%$ confidence levels of prestimulation interval. Note a transient suppression of spindles and ripples following HFS. Ripple probability is expectedly high in cases of the ripple-triggered stimulation. Log scale for ripple probability on $A$ is used for illustration purpose. (C) Post-stimulation ripple probability for RT-LFS (open bars) and RT-HFS (black bars). Solid line shows the interripple probability during nonstimulated condition; dashed lines indicate lower and upper 95\% confidence levels. (D) Interspindle interval during nonstimulated (dashed line) and HFS (solid line with filled circles) conditions. Inset shows interspindle interval for the LFS group. Note sleep spindle suppression during HFS.

stimulation the total time spent in the quiet awake state increased, while the total time spent in SWS decreased. Furthermore, the duration of each quiet awake and SWS episode was longer and shorter, respectively (quiet: $F_{(1,19)}=23.52, P<$ 0.001 ; SWS: $\left.F_{(1,19)}=11.33, P<0.01\right)$. However, for both variables there was neither a significant interaction nor between-group difference. The latter result indicated that these effects were not due to timing or frequency of LC stimulation, but could be attributed to another common factor such as, for example, maze exposure. Regrettably, the effect of learning experience per se could not be examined in the present study because no post-learning recordings were made without LC stimulation.

We next compared the occurrence of SWS-associated neural events such as delta waves, sleep spindles, and ripples between the baseline and LC-stimulation conditions. The ripple-triggered LFS or HFS suppressed occurrence of cortical high-amplitude delta waves (baseline versus LC-stimulation: $F_{(1,16)}=14.18, P<0.01$; interaction: $F_{(2,16)}=6.93, P<0.01$; Table 1). In contrast, random-HFS significantly reduced sleep spindles (baseline versus LC-stimulation: $F_{(1,17)}=10.36, P<0.01$; interaction: $F_{(2,17)}=$ $3.83, P<0.05$; Table 1$)$. None of the LC-stimulation conditions affected Delta index (see Methods) or ripple rate (Table 1).

Overall our results show that a learning experience combined with mild (not-awakening) phasic LC activation during the postlearning period prolongs the quiet awake state and shortens SWS. However, this shift in the awake/sleep pattern did not depend on the timing or frequency of LC stimulation and therefore could not cause the spatial memory deficit, seen exclusively in the RT-HFS group. This behavioral effect could be related to a higher general random LC activation was not effective. The low-frequency (20 $\mathrm{Hz}$ ) LC stimulation did not have behavioral effects or cause any disturbance in neural activity. We conclude that the memory deficit was due to interruption of critical hippocampal-cortical dialogue underlying off-line memory consolidation; however alternative interpretations are also plausible.

\section{Role of NE for synaptic and systems consolidation}

Our initial hypothesis was based on merging two distinct lines of research: synaptic and systems-level memory consolidation. First, it has been established by a large number of both in vivo and in vitro studies that NE release promotes or even permits long-term synaptic plasticity in the hippocampus (Hopkins and Johnston 1984; Gelinas and Nguyen 2005; Harley 2007; Reid and Harley 2010). The second line of research, more recent, establishes that experience-related neuronal ensemble replay occurs during SPW-Rs (Kudrimoti et al. 1999; Nadasdy et al. 1999). We assumed that phasic NE release coincident with reactivation of the hippocampal network representing new memories should lead to more efficient synaptic consolidation and therefore enhanced memory performance. In contrast to this prediction, post-learning RT-LFS had no effect on spatial task acquisition and RT-HFS caused a strong memory deficit. This result however does not exclude that NE-mediated modification of synaptic strength does take place off-line. The vast experimental evidence consistently shows that NE release in hippocampus creates a temporal window of heightened plasticity (Huerta and Lisman 1993; Katsuki et al. 1997; Reid and Harley 2010), but our current knowledge about 
Table 1. The effects of LC stimulation on awake/sleep pattern and sleep-associated neural activity

\begin{tabular}{|c|c|c|c|c|c|c|c|}
\hline & & \multicolumn{3}{|c|}{ Spontaneous Behavior } & \multicolumn{3}{|c|}{ Learning + post-learning LC stimulation } \\
\hline & & RT-LFS & RT-HFS & Random-HFS & RT-LFS & RT-HFS & Random-HFS \\
\hline \multirow[t]{3}{*}{ Total time, min } & Active awake & $19.19 \pm 3.80$ & $16.98 \pm 3.41$ & $13.88 \pm 3.69$ & $21.10 \pm 2.39$ & $21.99 \pm 1.51$ & $17.40 \pm 3.15$ \\
\hline & Quiet awake & $15.97 \pm 1.72$ & $18.38 \pm 2.93$ & $16.60 \pm 1.39$ & $23.08 \pm 2.07$ & $20.75 \pm 2.20$ & $25.09 \pm 3.09$ \\
\hline & Spindle-rich SWS & $22.07 \pm 2.22$ & $20.00 \pm 2.26$ & $27.69 \pm 2.90$ & $14.81 \pm 2.15$ & $14.29 \pm 2.15$ & $13.91 \pm 2.76$ \\
\hline \multirow[t]{2}{*}{ Episode time, min } & Quiet awake & $0.59 \pm 0.05$ & $0.54 \pm 0.05$ & $0.53 \pm 0.04$ & $0.93 \pm 0.11$ & $0.75 \pm 0.12$ & $0.89 \pm 0.12$ \\
\hline & Spindle-rich SWS & $0.76 \pm 0.12$ & $0.68 \pm 0.08$ & $0.83 \pm 0.09$ & $0.57 \pm 0.06$ & $0.53 \pm 0.08$ & $0.51 \pm 0.08$ \\
\hline \multirow[t]{4}{*}{ Spindle-rich SWS } & Delta index, au & $0.30 \pm 0.03$ & $0.30 \pm 0.02$ & $0.29 \pm 0.04$ & $0.27 \pm 0.03$ & $0.29 \pm 0.01$ & $0.33 \pm 0.03$ \\
\hline & Delta waves/min & $19.50 \pm 3.26$ & $15.57 \pm 2.93$ & $16.16 \pm 3.30$ & $9.50 \pm 2.65 \downarrow$ & $10.49 \pm 1.42 \downarrow$ & $17.38 \pm 3.26$ \\
\hline & Spindles/min & $15.27 \pm 0.97$ & $13.87 \pm 0.89$ & $16.50 \pm 1.01$ & $15.14 \pm 1.12$ & $11.77 \pm 1.72$ & $11.70 \pm 0.82 \downarrow$ \\
\hline & Ripples/min & $12.10 \pm 2.18$ & $19.20 \pm 2.78$ & $13.36 \pm 1.97$ & $9.53 \pm 1.36$ & $18.52 \pm 2.60$ & $10.99 \pm 3.12$ \\
\hline
\end{tabular}

Arrows indicate significant effects of LC stimulation (paired t-test, $\mathrm{p}<0.05$ ).

the temporal interaction between the synaptic and systems consolidation is rather incomplete. The results presented here point to a crucial importance of the timing of NE release. Earlier we have shown that LC neurons tend to fire during the transition from down-to-up state (Eschenko et al. 2012). Since ripples coincide with the cortical up states, this means that the LC burst is more likely to occur spontaneously before the ripple. An example of the importance of timing for NE action is found in NE-mediated facilitation of cortical responses to sensory stimulation. There, it is clear that priming stimulation of LC is most effective when applied 200-400 msec before the sensory stimulus presentation (Waterhouse et al. 1998).

At present, studies bridging the mechanisms of synaptic and systems-level of memory consolidation, particularly combined with testing animals on hippocampal-dependent learning tasks remain rather sparse. On one hand, the synaptic plasticity and memory hypothesis is supported by numerous demonstrations that bidirectional modifications of synaptic strength can be induced by stimulation mimicking physiological activity patterns such as theta-burst stimulation (Larson and Munkácsy 2015) or stimulation at the ripple-frequency range (Bliss and Lømo 1973). It has been shown in vitro that the stimulation protocols for LTP induction produce a pattern of physiological activity resembling SPW-Rs (Behrens et al. 2005). At the same time the experimental evidence remains controversial, as it has been recently reported that LTP was impaired in hippocampal slices that produce spontaneous SPW-Rs (Colgin et al. 2004). It remains yet to be demonstrated that the experience-induced selective modulation of synaptic strength takes place off-line and if it does, defining the time windows for synaptic plasticity and associated brain state appears to be critical.

The diffuse ascending neuromodulatory systems, and the LC-NE system being one of them, appear to be ideal candidates for bridging the cellular and systems-level mechanisms of memory consolidation. There is a substantial support for beneficial effects of NE for both online and off-line information processing (Sara et al. 1994; Roullet and Sara 1998; Sara 2000, 2009; Tronel et al. 2004). The $\beta$-adrenoreceptors in hippocampus facilitate LTP induction (Gelinas and Nguyen 2005) and also induction of SPW-Rs in vitro (Ul Haq et al. 2012); their activation during the post-learning period was critical for memory retrieval (Roullet and Sara 1998; Cahill et al. 2000). Novelty-induced phasic activation of LC-NE neurons was linked to increased neurotransmission in the dentate gyrus (Kitchigina et al. 1997); and LC stimulation prior to exposure to a novel environment facilitated LTD induction in CA1 and promoted encoding of spatial information (Lemon et al. 2009). To date, there is strong evidence for the critical impact of NE neurotransmission for synaptic consolidation, while a long-standing puzzle of engagement of the LC-NE system in the late phase of memory consolidation (Sara et al. 1999) remains yet to be resolved in future experiments.

Finally, any characteristic features of the SPW-Rs which would indicate replay of a specific neuronal assembly have not yet been identified. Ripples are commonly thought to indicate instances of sequential activation of hippocampal neurons, which is identical to the activity pattern driven by preceding experience (Skaggs and McNaughton 1996; Kudrimoti et al. 1999; Nadasdy et al. 1999). Recently, it has been suggested that the content of replay does not contain the traces of recent memories exclusively, but can also represent experiences more distant in time, as well as generate completely new activity patterns, which may represent results of information processing (Gupta et al. 2010; Singer et al. 2013; Wikenheiser and Redish 2015). In light of these recent revelations, it is not so surprising that unselective modification of synaptic strength by NE within functionally diverse cell assemblies activated during ripples was not favorable for stabilization of new memory traces.

\section{Hippocampal-cortical information transfer}

The SPW-R, which indicates a robust population burst in hippocampus, has been suggested to mediate information transfer from the hippocampus to neocortex for long-term storage (Buzsaki 1989) via functional coupling with slower-range oscillations in cortex (Diekelmann and Born 2010). Noradrenergic neuromodulation could, in principle, facilitate such hippocampalcortical interaction. Activation of LC-NE neurons and associated NE release has been shown to increase synaptically driven activity in many brain regions including hippocampus and cortex (Segal and Bloom 1976; Hasselmo et al. 1997; Devilbiss and Waterhouse 2004). Earlier findings are in agreement with more recent studies showing that phasic LC activation transiently shifts excitatory/inhibitory balance toward excitation in the ventral subiculum (Lipski and Grace 2013) and in the prefrontal cortex (Marzo et al. 2014). Thus, simultaneous NE release could rapidly bring cortex and hippocampus to a more excitable (Up) state and therefore facilitate cross-regional interaction, for example, by phase-resetting of ongoing slow waves (Eschenko et al. 2012). In contrast, the ripple-associated phasic LC activation introduced interference in the hippocampal-cortical communication likely by decoupling hippocampal ripples and cortical spindles.

The coordinated ripple/spindle occurrence has been suggested to mediate the hippocampal-cortical information transfer underlying memory consolidation (Siapas and Wilson 1998). In the present study, RT-HFS delivered to the neuromodulatory center did not disrupt ongoing ripple, but phasic NE release within thalamo-cortical network made spindle generation transiently 
impossible; therefore temporarily precise co-occurrence of ripples and spindles has been practically eliminated during the 1-h postlearning period. Two previous studies, which used electrical stimulation of the ventral hippocampal commissure at times of ripple onset leading to the disruption of SPW-Rs, reported suppression of neuronal firing in CA1 for about $1 \mathrm{sec}$ after stimulation (Girardeau et al. 2009; Ego-Stengel and Wilson 2010), while the cortical activity appeared to be unaffected (Girardeau et al. 2009). Yet, it remains to be established if a memory deficit was attributed to the interruption of ripple-associated replay or to disturbance of hippocampal-cortical communication. Our findings, not refuting the critical role of the ripple-associated neuronal replay in hippocampus, provide further support to the hypothesis about the essential role of coordinated interaction between hippocampus and frontal cortex, as reflected for example by ripple-spindle coupling, for faithful storage of newly acquired information.

\section{Competing functional networks}

The interference in hippocampal-cortical communication and associated memory deficit could be caused by LC stimulation induced activation of a competing large-scale functional network. Our recent fMRI study showed that many cortical areas were activated during ripples, while the LC-along with other neuromodulatory centers and a number of subcortical brain regions-was inhibited (Logothetis et al. 2012). This striking activation/deactivation pattern suggests that the LC may belong to a functionally distinct large-scale network, which is suppressed during the time windows of hippocampal-cortical communication. The septalhippocampal circuit, which supports exploratory and attentive behaviors (Vertes and Kocsis 1997), could be one of the competing networks. It has been shown that firing of the medial septal neurons is suppressed around ripples (Dragoi et al. 1999; Vandecasteele et al. 2014); in turn, activation of the septo-hippocampal pathway suppresses ripple generation (Vandecasteele et al. 2014). The medial septum is a major hippocampal afferent and both structures are heavily innervated by NE fibers (Loy et al. 1980). Activation of LC-NE neurons or increased NE neurotransmission within septum induces theta rhythmicity of the medial septal neurons and augments theta-oscillatory activity in hippocampus (Berridge and Foote 1991; Berridge et al. 1996; Hajos et al. 2003; Kitchigina et al. 2003; Brown et al. 2005). Thus, there appear antagonistic relationships between the septohippocampal and the ripple-generation networks. Increased NE neurotransmission clearly reinforces the septo-hippocampal pathway, while the effects of NE on the hippocampal-cortical functional interaction are not yet sufficiently investigated.

The thalamo-cortical network is another candidate for competing network which could be potentiated by NE release. We have recently shown that electrical stimulation of LC transiently suppressed delta waves and spindle-range oscillations in the prefrontal cortex in urethane-anesthetized rat (Marzo et al. 2014), possibly by a NE-mediated switch from bursting to tonic mode of firing of thalamic neurons. It is well documented that NE input shifts the thalamo-cortical network from a synchronized state associated with delta waves and spindles to a desynchronized state characterized by increased neuronal responsiveness to synaptic inputs, which is more optimal for sensory processing (McCormick 1989). Our preliminary results in naturally sleeping rats indicated that $\mathrm{LC}$ stimulation parameters similar to the ones used in the present study produce a transient increase in gamma power and firing probability in sensory and associative thalamic nuclei (unpublished observations).

The competing networks hypothesis is further supported by the results of a recent study where ongoing neural activity during the intertrial interval was interrupted by bright flashes of light at times of ripple occurrence in the rabbit (Nokia et al. 2012). Salient sensory stimulation in any modality elicits a burst of LC-NE neurons (Foote et al. 1980; Herve-Minvielle and Sara 1995). In this case, the visual stimulus presented at ripple onset transiently shifted hippocampal activity to the theta-oscillation state and caused impaired acquisition of a trace eye blink conditioning, a hippocampus-dependent associative learning task (Nokia et al. 2012). Clearly, introducing any interference to the post-learning information processing either by disruption of activity within the supporting network or by coactivation of competing networks would lead to less efficient consolidation.

\section{Conclusion}

The results reported here did not support our original prediction that pairing of LC stimulation with SPW-Rs would potentiate synaptic consolidation within the putative experience-activated hippocampal-cortical network. The negative effect on memory of the high-frequency LC stimulation does not, in any way, question the role of the NE neurotransmission in promoting synaptic consolidation, for which there is unequivocal evidence at a cellular, synaptic, and behavioral level across species (Harley 2007; Sara 2009 , 2015). Instead, our findings bring into question the timing of NE release for synaptic and systems-level memory consolidation. In addition, we have shown that the memory deficit was accompanied by decoupling of coordinated hippocampal-cortical activity due to LC activation; the latter finding emphasizes the role of the hippocampal-cortical dialogue during off-line consolidation and merits continued investigation.

\section{Materials and Methods}

\section{Animals}

Forty-eight male Sprague-Dawley rats (initial weight 200-250 g, Charles River Laboratory, Germany) were used in the present study. Rats were housed in groups of four and had food and water access ad libitum on a $12 \mathrm{~h}$ light-dark cycle (8:00 a.m. lights on). During 2 -wk of training on a maze rats were maintained at $\sim 80 \%$ of ad libitum weight via restricted food intake ( $\sim 15 \mathrm{~g}$ per rat $/ 24 \mathrm{~h}$; weight was monitored daily). Experiments were approved by the local authorities (Regierungspräsidium Tübingen, Germany, Referat 35, Veterinärwesen), in accordance with the regional animal welfare committee pursuant to $\$ 15$ of the German Animal Welfare Act (Kommission nach $\S 15$ des Tierschutzgesetzes), and were in full compliance with the Directive 2010/63/EU of the European Parliament and of the council on the protection of animals used for scientific purposes.

\section{Behavioral apparatus}

Rats were tested on an elevated ( $84 \mathrm{~cm}$ above the floor) eight-arm radial maze. Black aluminum alleys $(66 \mathrm{~cm} \times 10 \mathrm{~cm})$ extended from a center platform (diameter $30 \mathrm{~cm}$ ); at the end of each maze alley a small aluminum cup (diameter $6 \mathrm{~cm}$, depth $1 \mathrm{~cm}$ ) could contain a reward ( $0.15 \mathrm{~g}$ piece of Kellogg's cereal). A few extra-maze visual cues were available in a dimly lit experimental room. The training procedure required presence of the experimenter in the room during the duration of the training session. During the trial, the experimenter remained still and in a fixed position relative to the maze.

\section{Habituation and pretraining}

The rats were first familiarized with the food used as reward (Kellogg's cereal) in their home cages and were gently handled daily by the experimenter. Twenty-four hours before the first exposure to the maze the food deprivation started and the animals were maintained on a restricted food schedule $(15 \mathrm{~g} / 24 \mathrm{~h})$ until the end of the learning experiment. For the first habituation session, reward pellets were evenly distributed across the entire 
maze surface. A rat was placed on the central platform and was allowed to freely explore the maze for $25 \mathrm{~min}$. If an animal showed freezing behavior or any signs of stress, the session was interrupted and repeated next day. In subsequent habituation sessions, the location of reward pellets was gradually moved toward the end of the maze alleys. The habituation sessions continued for $2-3 \mathrm{~d}$ until the rat expressed reward-motivated behavior, easily retrieved reward from the cups, and readily consumed it. For the pretraining session, all reward cups were baited with three pellets. The rat was placed on the central platform and allowed to retrieve the rewards. The pretraining session ended when the animal either collected all rewards or after $20 \mathrm{~min}$. In the latter case, the pretraining session was repeated on the following days until the rat was able to collect all available rewards within $20 \mathrm{~min}$.

\section{Spatial discrimination task}

For the spatial discrimination task, three out of eight maze arms were baited with reward to minimize working memory load, while maintaining spatial (reference) memory demand for successful task performance. The spatial configuration of the three baited maze arms was randomly assigned for each rat and kept the same for all eight learning sessions. In the beginning of each trial, the rat was placed on the center platform and had access to all eight maze arms. The trial continued until the reward from all three baited maze arms was collected and consumed or $5 \mathrm{~min}$ elapsed. Each daily learning session consisted of three trials. Between trials, the rat was placed into a nontransparent small Plexiglas cage for $\sim 1 \mathrm{~min}$. During the intertrial interval the maze was wiped in order to remove olfactory cues and re-baited. For each rat, the learning sessions took place at the same time of the day.

The following behavioral variables were registered in each trial: (1) total number of maze arms visited (a maze arm entry was registered when a rat left the central platform with all four limbs); (2) number of reference memory errors (entries into unbaited maze arms), (3) number of working memory errors (reentries into visited maze arms), and (4) trial time (time required to collect and consume all three rewards). In addition, the performance accuracy was calculated as a proportion of visits to the baited maze arms of total number of maze arm entries. The session Mean values were calculated over the three daily trials for statistical analysis. Rats underwent electrode implantation surgery before learning.

\section{Surgery and electrode placement}

Surgery was performed under isoflurane anesthesia (initiation $\sim 4 \%$, maintenance $\sim 1.6 \%$ ). Depth of anesthesia was monitored by ensuring lack of responses to mildly noxious stimuli (pinch of hind paw). Heart rate and blood oxygenation were monitored using a pulse oximeter (Nonin 8600V, Nonin Medical, Inc.) and supplementary oxygen was provided to maintain the blood oxygenation level above $90 \%$. Body temperature was maintained at $37^{\circ} \mathrm{C}$ throughout the surgery. A fully anesthetized rat was fixed in a stereotaxic frame (David Kopf Instruments). The head angle was set at zero degrees. Local anesthetic (Lidocard 2\%, B. Braun) was applied to the exposed skull and skin surface. Burr holes for the electrodes and fixation screws were made. Stainless steel screws served as anchor, electrode, and ground. The screw electrode for EEG recording was placed above the frontal cortex; the ground/reference screw was placed above the posterior cortex; the ground screw for microstimulation was placed above cerebellum. Screws were fixed in the skull and additionally secured with tissue adhesive.

For simultaneous recording in cortex and hippocampus in-house developed and constructed linear electrode arrays were used. Briefly, eight Pt/Ir (90/10) wires (7 $\mu \mathrm{m}$ diameter; California Fine Wire) were embedded and glued into a metal electrode carrier with $200 \mu \mathrm{m}$ spacing between the recording sites. The impedance of each electrode contact was adjusted to $\sim 0.3 \mathrm{M} \Omega$. For LC stimulation, we used a single Pt/Ir electrode with impedance of $0.3-0.5 \mathrm{M} \Omega$ (FHC, Bowdoin ME, USA).
Linear electrode arrays and the stimulation electrode were placed in the target brain areas using a high-precision stereotaxic micromanipulator (David Kopf Instruments). The final adjustment of the electrode position was guided by online monitoring of neural activity. The HPC/CTX electrode was implanted at $\sim 3 \mathrm{~mm}$ from the brain surface such that the deep electrode contacts reach the CA1 pyramidal cell layer and the upper electrode contacts remain within somatosensory cortex above the dorsal hippocampus (AP: -3.0 , from bregma and L: 2.5, from midline). The LC electrode was implanted at $\sim 15^{\circ}$ angle using the following coordinates: $\mathrm{AP}=-4.0-4.2$ (from lambda); $\mathrm{L}=1.0-1.2 ; \mathrm{DV}: 5.5-6.2 \mathrm{~mm}$. The accuracy of LC targeting was verified using electrophysiological recording. The following criteria were used for identification of LC neurons: (1) broad spike widths $(\sim 0.6 \mathrm{msec}),(2)$ regular low firing rate (1-2 spikes/s), and (3) a brief excitation followed by prolonged inhibition in response to pinch of the contralateral paw (Cedarbaum JM, Aghajanian GK. 1978). Once the electrode position was optimized, the surgery was completed by fixating the electrodes and the anchor screws to the skull using dental cement (Paladur, Heraeus Kulzer $\mathrm{GmbH}$ ). The injection of analgesic $(2.5 \mathrm{mg} / \mathrm{kg}$, s.c; Finadyne, Essex) and antibiotic $(5.0 \mathrm{mg} / \mathrm{kg}$, s.c.; Baytril, Bayer) was given before rat awakening from anesthesia and repeated at $24 \mathrm{~h}$ intervals during next $4 \mathrm{~d}$. The animals were allowed 5-7 d for post-surgical recovery when food and water were present at libitum.

\section{Electrophysiological recording and electrical stimulation}

After post-surgical recovery, each rat was habituated to the recording/sleep box and the cable connection procedure. Recording was performed in a nontransparent Plexiglas cage $(30 \times 20 \times 20 \mathrm{~cm})$ placed in a shielded metal box with $50 \mathrm{~cm}$ walls. The recording setup was located in a quiet, isolated room adjacent to the room with the maze where the learning experiment was carried out. The rat was connected to the amplifier by a cable allowing free movement within the sleep box. For recording of the extracellular local field potentials (LFPs), the signals were high-pass $(0.1 \mathrm{~Hz})$ filtered and amplified $(\times 25)$ using an in-house built preamplifier, then low-pass $(300 \mathrm{~Hz})$ filtered and amplified $(\times 1000)$ using a multichannel amplifier (MPC Plus, Alpha Omega Co.), and digitized at $5 \mathrm{kHz}$ using analog-to-digital converter Power1401mkll (CED). The rat behavior was tracked by a video camera (Quickcam, Logitech) mounted above the sleep box. The video and electrophysiological recordings were synchronized using the Spike2 software (CED). The recordings were made between 10 a.m. and 8 p.m. during the light phase of the light-dark period.

Monopolar electrical stimulation was applied unilaterally to the LC using trains of bi-phasic (cathodal leading) square pulses $(0.4 \mathrm{msec}, 0.05 \mathrm{~mA}$ ) presented at $20-100 \mathrm{~Hz}$ for $100-200 \mathrm{msec}$. Based on existing knowledge and our own empirical data, $0.4 \mathrm{msec}$ proved to be the most efficient pulse-duration for activating unmyelinated nerve fibers. We used current intensities up to $0.05 \mathrm{~mA}$, the repetitive use of which did not cause any local tissue damage. According to our previous study, monopolar stimulation with this current intensity reliably activated LC-NE neurons within a radius of $0.2 \mathrm{~mm}$ and also elicited phasic activation in the contralateral LC (Marzo et al. 2014). The stimulation parameters were digitally controlled by the Spike2 software (CED) and transmitted to the in-house built current source via digitalto-analog converter Power 1401mkII (CED). The voltage passed through the tip of the stimulation electrode was monitored via a custom-designed voltage output unit. Prior to the learning experiment, the effectiveness of LC stimulation was tested in each rat by applying intermittent (8-11-sec interstimulus interval) LC stimulation with variable pulse frequency $(20-200 \mathrm{~Hz})$ and train duration (100-500 msec) during spontaneous behavior. Shortly after placing them in a familiar sleep-box, rats commonly calmed down, curled up, and fell asleep. The animal state was continuously monitored online. The stimulation was applied when the animal was in a quiet awake state or asleep and was discontinued when the rat awoke or was engaged in grooming or exploration. None of the stimulation parameters used here elicited jaw or facial twitching, which suggests that the effective voltage gradient 
produced by the stimulation current was likely concentrated around the LC cell bodies and did not spread beyond the LC and activate the adjacent trigeminal pathway.

The stimulation parameters, producing behavioral arousal or awakening from sleep, were defined as "threshold" and were not used in the main experiments during post-learning sessions (Supplemental Fig. 1A). The LC stimulation applied for more than $200 \mathrm{msec}$ often caused the rat to wake up. The low-frequency LC stimulation (LFS, $0.05 \mathrm{~mA}, 20 \mathrm{~Hz}, 200 \mathrm{msec}$ ) has never produced any noticeable behavioral responses or caused detectable changes in EEG (Fig. 2B, left panel). The high-frequency LC stimulation (HFS, $0.05 \mathrm{~mA}, 50 / 100 \mathrm{~Hz}, 100 / 200 \mathrm{msec}$ ) was subthreshold for behavioral arousal (Supplemental Fig. 1B), but consistently produced a transient cortical desynchronization (i.e., power increase in high-frequencies and decrease in lowfrequencies), when applied during sleep (Fig. 2B, right panel). In some cases, none of the stimulation parameters elicited either behavioral or neural response. The post hoc histological examination confirmed that in seven out of eight such cases, the electrode tip was found outside of the LC core; the one "noneffective" case was likely due to some technical failure, for example, inability to effectively pass electrical current via the electrode tip.

Once the subthreshold stimulation parameter was established "on-line" based on the experimenter's visual observation, the effectiveness and reliability of LC stimulation was additionally verified "off-line". To this end, the data obtained for different stimulation parameters during the test/calibration sessions were submitted to the band-limited power (BLP) analysis described in detail elsewhere (Marzo et al. 2014). Briefly, the cortical EEG/ LFP signal was band-pass filtered at different frequency ranges and the BLP was computed as the square of the absolute value of the Hilbert transform of the band-passed signal of each band. Next, the BLP for each frequency range was normalized to 2-sec prestimulus interval ( $z$-score) and plotted -2 to $4 \mathrm{sec}$ around the onset of LC stimulation (Fig. 2B). A color-coded scale of the BLP changes readily revealed the "effective" stimulation, which typically produced power decrease within low frequencies $(<30 \mathrm{~Hz})$ and power increase in the higher frequencies $(>30 \mathrm{~Hz})$. Up to three calibration recordings were required to establish the optimal stimulation parameters.

A nonimplanted control was trained on a maze along with the implanted groups and rats were left undisturbed in their home cages after each training session. The implanted rats were assigned to one of three stimulation conditions. The microstimulation was applied either randomly or at the onset of a ripple event detected "online" by crossing of a threshold on the band-pass (140-240) filtered CA1 LFPs. The threshold crossing automatically triggered a train of electric pulses delivered to the LC (Fig. 1). One group received ripple-triggered LFS (RT-LFS, $n=7$ ), which was below awakening threshold and did not cause detectable change in EEG (Fig. 2B, left panel). In all rats assigned to this group, the higher-frequency stimulation did cause transient cortical activation during calibration sessions. The other two groups received (nonawakening) HFS either during ripples (RT-HFS, $n=7$ ) or randomly with 2-11-sec interstimulus intervals (Random-HFS, $n=8$ ). The main experiment began with a baseline recording session when neither maze exposure nor LC stimulation took place. The rat was transported from the housing facility, connected to the recording cable, and spontaneous behavior was monitored for $1 \mathrm{~h}$.

\section{Electrophysiological signal processing and detection of neural events}

Electrophysiological data processing was performed using the Spike2 software (CED) and custom software written in Spike2 script language and in MATLAB (The MathWorks).

Behavioral state during the 1 -h recording session was determined by a semiautomated scoring algorithm based on a combination of indices extracted from cortical EEG channel and video monitored movement. The artifact-free EEG/LFP signal was bandpass filtered and split into different frequency bands: delta $(\delta ; 1-$ $4 \mathrm{~Hz})$, theta $(\theta ; 4-8 \mathrm{~Hz})$, sigma $(\sigma ; 12-16 \mathrm{~Hz})$, and gamma $(\gamma ; 45-$
$90 \mathrm{~Hz}$ ). The following indices were calculated using band-limited power values: Delta index $(\mathrm{D}-\mathrm{idx}=\delta / \gamma)$; Theta/Delta ratio $(\mathrm{T} / \mathrm{D}=$ $\theta / \delta)$; non-REM sleep index $($ NREM-idx $=(\delta \times \sigma) / \gamma)$; REM sleep in$\operatorname{dex}\left(\operatorname{REM}-i d x=\theta^{2} /(\delta \times \sigma)\right)$; quiet awake index $(\mathrm{QW}$-idx $=\gamma /(\delta \times$ $\sigma)$ ). For each index, a threshold was determined by an expert observer and kept identical for all recordings obtained from the same rat. Each of the 10-sec recording epochs was automatically assigned to one of the six behavioral states according to the following algorithm: (1) "active awake state" was defined by the presence of locomotor activity (decoded from video), rearing, or grooming; (2) "quiet awake state" was defined by the absence of locomotor activity, high (suprathreshold) QW-idx, high T/D, low (subthreshold) D-inx, and low NREM-idx; (3) "spindle-rich SWS" was identified by high D-idx, high NREM-idx, low T/D, and presence of at least one sleep spindle per 10-sec epoch; (4) "deep SWS" was identified similar to "spindle-rich SWS" except for absence of sleep spindles; (5) "pre-REM sleep" was scored by declining D-idx, rising T/D, and presence of high-amplitude fast sleep spindles; (6) "REM sleep" was identified by low D-idx, high $\mathrm{T} / \mathrm{D}$, and high REM-idx. The behavioral states were additionally verified by an expert observer with the help of video recording. The state-dependent EEG power spectrum is shown on Supplemental Figure 3.

The "sleep spindles" were automatically detected from the artifact-free cortical EEG/LFP channel by band-pass $(\sigma ; 12-16$ $\mathrm{Hz}$ ) filtering, down-sampling to $200 \mathrm{~Hz}$, and thresholding of the $\sigma$-band power envelope (root mean square with 0.2 -sec smoothing; $\sigma$-RMS). The spindle detection threshold was calculated as 2 SDs of the $\sigma$-RMS during baseline (no LC-stimulation) spindle-rich SWS and kept constant for all recording sessions obtained from the same rat. Minimal sleep spindle length was defined as $0.3 \mathrm{sec}$ of continuous $\sigma$-RMS values above the threshold. The high-amplitude "delta waves" were detected from the artifactfree cortical EEG/LFP channel by low-pass $(\delta ; 4 \mathrm{~Hz})$ filtering and down-sampling to $100 \mathrm{~Hz}$. Delta wave cycles with a maximal amplitude of at least $-100 \mu \mathrm{V}$ for EEG and $-400 \mu \mathrm{V}$ for LFPs and with at least $0.16 \mathrm{sec}$ between zero crossings were selected. For detection of "hippocampal ripples," the LFP signal recorded from the CA1 subfield was band-pass $(120-240 \mathrm{~Hz})$ filtered and the signal power was calculated (root mean square with time constant $0.02 \mathrm{sec}$, ripple-RMS). The ripple event was detected when the ripple-RMS signal exceeded a 4SDs threshold of the mean ripple-RMS. The start and the end of a ripple event were marked by 2 SDs thresholding of the ripple-RMS signal if these two time points were separated by at least $0.02 \mathrm{sec}$. For all detected events, the mean event rate (events/min) was calculated for each behavioral state over entire recording session.

\section{Statistical analysis}

Behavioral and neural data were evaluated by using various designs of analysis of variance (ANOVA) and post hoc comparisons when appropriate. For the repeated-measures design the day of training was used as a within-subject (repeated) factor and the LC stimulation parameter as a between-subject (group) factor. The statistical significance $\alpha$ value was set at $P<0.05$ level. The IBM SPSS Statistics (v.22) software package was used for statistical analysis.

\section{Perfusion and Histology}

The electrode placement was additionally verified by histological examination. At the end of the experiment, the rat was euthanized with a lethal dose of sodium pentobarbital $(100 \mathrm{mg} / \mathrm{kg}$ i.p.; Narcoren, Merial $\mathrm{GmbH}$ ) and perfused transcardially with $0.9 \%$ saline followed by $4 \%$ paraformaldehyde in $0.1 \mathrm{M}$ phosphate buffer (PB, pH 7.4). The brain was removed and stored in the same fixative. Before sectioning, the whole brain was placed in $0.1 \mathrm{M} \mathrm{PB}$ buffer containing 30\% sucrose until it sank. Serial $60-\mu \mathrm{m}$-thick coronal sections were cut on a horizontal freezing microtome (Microm HM 440E, Walldorf), collected in $0.1 \mathrm{M} \mathrm{PB}$ and then directly stained or stored at $-20^{\circ} \mathrm{C}$ in a cryoprotectant solution (30\% ethylene glycol and 10\% sucrose in $0.05 \mathrm{M} \mathrm{PB}$ ) until further 
processing. The Nissl staining was made following a standard procedure. Briefly, sections were mounted onto gelatin-coated glass slides, defatted, stained with Cresyl violet, rinsed with acetic acid, dehydrated, and cover-slipped. All sections were examined under bright field using an AxioPhot or Axiolmager microscope (Carl Zeiss, Goettingen). Position of the electrode tip relative to LC and HPC was assessed visually and digitized.

\section{Acknowledgments}

We thank Nelson Totah and Masataka Watanabe for valuable comments on the manuscript; Katalin Kalya for help with behavioral experiments and histology, Sanja Stegerer for building electrode arrays, Geoff Horseman for providing sleep scoring software, Eduard Krampe, Axel Oeltermann, and Joachim Werner for technical support. This work was partially supported by the SI-CODE project of the Future and Emerging Technologies (FET) programme within the 7th Framework Programme for Research of the European Commission, under FET-Open grant number: FP7-284553 (Y.N., N.K.L., and O.E.) and by the German Academic Exchange Service (DAAD), grant number: A/10/85843 (Y.N.).

\section{References}

Aston-Jones G, Bloom FE. 1981. Norepinephrine-containing locus coeruleus neurons in behaving rats exhibit pronounced responses to non-noxious environmental stimuli. J Neurosci 1: 887-900.

Bailey CH, Giustetto M, Huang Y-Y, Hawkins RD, Kandel ER. 2000. Is heterosynaptic modulation essential for stabilizing Hebbian plasticity and memory?. Nat Rev Neurosci 1: 11-20.

Battaglia FP, Sutherland GR, McNaughton BL. 2004. Hippocampal sharp wave bursts coincide with neocortical "up-state" transitions. Learn Mem 11: $697-704$

Battaglia FP, Benchenane K, Sirota A, Pennartz CM, Wiener SI. 2011. The hippocampus: hub of brain network communication for memory. Trends Cogn Sci 15: 310-318.

Behrens CJ, van den Boom LP, de Hoz L, Friedman A, Heinemann U. 2005 Induction of sharp wave-ripple complexes in vitro and reorganization of hippocampal networks. Nat Neurosci 8: 1560-1567.

Berridge CW, Foote SL. 1991. Effects of locus coeruleus activation on electroencephalographic activity in neocortex and hippocampus. I Neurosci 11: $3135-3145$

Berridge CW, Bolen SJ, Manley MS, Foote SL. 1996. Modulation of forebrain electroencephalographic activity in halothane-anesthetized rat via actions of noradrenergic $\beta$-receptors within the medial septal region. I Neurosci 16: 7010-7020.

Bliss TVP, Lømo T. 1973. Long-lasting potentiation of synaptic transmission in the dentate area of the anaesthetized rabbit following stimulation of the perforant path. J Physiol 232: 331-356.

Brown RAM, Walling SG, Milway JS, Harley CW. 2005. Locus ceruleus activation suppresses feedforward interneurons and reduces $\beta-\gamma$ electroencephalogram frequencies while it enhances $\theta$ frequencies in rat dentate gyrus. J Neurosci 25: 1985-1991.

Buzsaki G. 1985. What does the LTP model of memory model? In Brain plasticity, learning, and memory (ed. Will B, Schmitt $\mathrm{P}$, Dalrymple-Alford JC), pp. 157-166. Plenum Press, New York.

Buzsaki G. 1989. Two-stage model of memory trace formation: a role for "noisy" brain states. Neuroscience 31: 551-570.

Cahill L, Pham CA, Setlow B. 2000. Impaired memory consolidation in rats produced with $\beta$-adrenergic blockade. Neurobiol Learn Mem 74: $259-266$.

Colgin LL, Kubota D, Jia Y, Rex CS, Lynch G. 2004. Long-term potentiation is impaired in rat hippocampal slices that produce spontaneous sharp waves. I Physiol 558: $953-961$.

Devilbiss DM, Waterhouse BD. 2004. The effects of tonic locus ceruleus output on sensory-evoked responses of ventral posterior medial thalamic and barrel field cortical neurons in the awake rat. J Neurosci 24: 10773-10785.

Diekelmann S, Born J. 2010. The memory function of sleep. Nat Rev 11: $114-126$.

Dragoi G, Carpi D, Recce M, Csicsvari J, Buzsáki G. 1999. Interactions between hippocampus and medial septum during sharp waves and $\theta$ oscillation in the behaving rat. J Neurosci 19: 6191-6199.

Ego-Stengel V, Wilson MA. 2010. Disruption of ripple-associated hippocampal activity during rest impairs spatial learning in the rat. Hippocampus 20: $1-10$.
Eschenko O, Sara SJ. 2008. Learning-dependent, transient increase of activity in noradrenergic neurons of locus coeruleus during slow wave sleep in the rat: brain stem-cortex interplay for memory consolidation? Cereb Cortex 18: 2596-2603.

Eschenko O, Ramadan W, Molle M, Born J, Sara SJ. 2008. Sustained increase in hippocampal sharp-wave ripple activity during slow-wave sleep after learning. Learn Mem 15: 222-228.

Eschenko O, Magri C, Panzeri S, Sara SJ. 2012. Noradrenergic neurons of the locus coeruleus are phase locked to cortical up-down states during sleep. Cereb Cortex 22: 426-435.

Florin-Lechner SM, Druhan JP, Aston-Jones G, Valentino RJ. 1996. Enhanced norepinephrine release in prefrontal cortex with burst stimulation of the locus coeruleus. Brain Res 742: 89-97.

Foote SL, Aston-Jones G, Bloom FE. 1980. Impulse activity of locus coeruleus neurons in awake rats and monkeys is a function of sensory stimulation and arousal. Proc Natl Acad Sci 77: 3033-3037.

Gelinas JN, Nguyen PV. 2005. $\beta$-adrenergic receptor activation facilitates induction of a protein synthesis-dependent late phase of long-term potentiation. J Neurosci 25: 3294-3303.

Girardeau G, Benchenane K, Wiener SI, Buzsaki G, Zugaro MB. 2009 Selective suppression of hippocampal ripples impairs spatial memory. Nat Neurosci 12: 1222-1223.

Gupta AS, van der Meer MA, Touretzky DS, Redish AD. 2010. Hippocampal replay is not a simple function of experience. Neuron 65: 695-705.

Hajos M, Hoffmann WE, Robinson DD, Yu JH, Hajos-Korcsok E. 2003. Norepinephrine but not serotonin reuptake inhibitors enhance $\theta$ and $\gamma$ activity of the septo-hippocampal system. Neuropsychopharmacology 28: $857-864$

Harley CW. 2007. Norepinephrine and the dentate gyrus. In Progress in brain research (ed. Helen ES), Vol. 163, pp. 299-318. Elsevier.

Harley CW, Sara SJ. 1992. Locus coeruleus bursts induced by glutamate trigger delayed perforant path spike amplitude potentiation in the dentate gyrus. Exp Brain Res 89: 581-587.

Hasselmo ME, Linster C, Patil M, Ma D, Cekic M. 1997. Noradrenergic suppression of synaptic transmission may influence cortical signal-to-noise ratio. J Neurophysiol 77: 3326-3339.

Herve-Minvielle A, Sara SJ. 1995. Rapid habituation of auditory responses of locus coeruleus cells in anaesthetized and awake rats. Neuroreport 6: $1363-1368$.

Hopkins WF, Johnston D. 1984. Frequency-dependent noradrenergic modulation of long-term potentiation in the hippocampus. Science 226: $350-352$

Huerta PT, Lisman JE. 1993. Heightened synaptic plasticity of hippocampal CA1 neurons during a cholinergically induced rhythmic state. Nature 364: $723-725$.

Katsuki H, Izumi Y, Zorumski CF. 1997. Noradrenergic regulation of synaptic plasticity in the hippocampal CA1 region. J Neurophysiol 77: 3013-3020.

King C, Henze DA, Leinekugel X, Buzsáki G. 1999. Hebbian modification of a hippocampal population pattern in the rat. J Physiol 521: 159-167.

Kitchigina V, Vankov A, Harley C, Sara SJ. 1997. Novelty-elicited, noradrenaline-dependent enhancement of excitability in the dentate gyrus. Eur J Neurosci 9: 41-47.

Kitchigina VF, Kutyreva EV, Brazhnik ES. 2003. Modulation of $\theta$ rhythmicity in the medial septal neurons and the hippocampal electroencephalogram in the awake rabbit via actions at noradrenergic

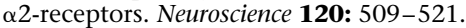

Kobayashi K, Yasoshima Y. 2001. The central noradrenaline system and memory consolidation. Neuroscientist 7: 371-376.

Kudrimoti HS, Barnes CA, McNaughton BL. 1999. Reactivation of hippocampal cell assemblies: effects of behavioral state, experience, and EEG dynamics. J Neurosci 19: 4090-4101.

Larson J, Munkácsy E. 2015. Theta-burst LTP. Brain Res 1621: 38-50.

Lemon N, Aydin-Abidin S, Funke K, Manahan-Vaughan D. 2009. Locus coeruleus activation facilitates memory encoding and induces hippocampal LTD that depends on $\beta$-adrenergic receptor activation. Cereb Cortex 19: 2827-2837.

Lipski WJ, Grace AA. 2013. Activation and inhibition of neurons in the hippocampal ventral subiculum by norepinephrine and locus coeruleus stimulation. Neuropsychopharmacology 38: 285-292.

Logothetis NK, Eschenko O, Murayama Y, Augath M, Steudel T, Evrard HC, Besserve M, Oeltermann A. 2012. Hippocampal-cortical interaction during periods of subcortical silence. Nature 491: 547-553.

Loy R, Koziell DA, Lindsey JD, Moore RY. 1980. Noradrenergic innervation of the adult rat hippocampal formation. J Comp Neurol 189: 699-710.

Marr D. 1971. Simple memory: a theory for archicortex. Philos Trans $R$ Soc Lond B Biol Sci 262: 23-81.

Marzo A, Totah NK, Neves RM, Logothetis NK, Eschenko O. 2014. Unilateral electrical stimulation of rat locus coeruleus elicits bilateral response of norepinephrine neurons and sustained activation of medial prefrontal cortex. J Neurophysiol 111: 2570-2588. 
McCormick DA. 1989. Cholinergic and noradrenergic modulation of thalamocortical processing. Trends Neurosci 12: 215-221.

McGaugh JL. 2013. Making lasting memories: remembering the significant. Proc Natl Acad Sci 110: 10402-10407.

Molle M, Yeshenko O, Marshall L, Sara SJ, Born J. 2006. Hippocampal sharp wave-ripples linked to slow oscillations in rat slow-wave sleep. I Neurophysiol 96: 62-70.

Nadasdy Z, Hirase H, Czurko A, Csicsvari J, Buzsaki G. 1999. Replay and time compression of recurring spike sequences in the hippocampus. J Neurosci 19: 9497-9507.

Nokia MS, Mikkonen JE, Penttonen M, Wikgren J. 2012. Disrupting neural activity related to awake-state sharp wave-ripple complexes prevents hippocampal learning. Front Behav Neurosci 6: 84.

Pavlides C, Winson J. 1989. Influences of hippocampal place cell firing in the awake state on the activity of these cells during subsequent sleep episodes. J Neurosci 9: 2907-2918.

Pennartz CM, Uylings HB, Barnes CA, McNaughton BL. 2002. Memory reactivation and consolidation during sleep: from cellular mechanisms to human performance. Prog Brain Res 138: 143-166.

Ramadan W, Eschenko O, Sara SJ. 2009. Hippocampal sharp wave/ripples during sleep for consolidation of associative memory. PLoS One 4: e6697.

Reid A, Harley C. 2010. An associativity requirement for locus coeruleus-induced long-term potentiation in the dentate gyrus of the urethane-anesthetized rat. Exp Brain Res 200: 151-159.

Roullet P, Sara S. 1998. Consolidation of memory after its reactivation: involvement of $\beta$ noradrenergic receptors in the late phase. Neural Plast 6: $63-68$.

Saar D, Barkai E. 2003. Long-term modifications in intrinsic neuronal properties and rule learning in rats. Eur J Neurosci 17: 2727-2734.

Sara SJ. 2000. Retrieval and reconsolidation: toward a neurobiology of remembering. Learn Mem 7: 73-84.

Sara SJ. 2009. The locus coeruleus and noradrenergic modulation of cognition. Nat Rev 10: 211-223.

Sara SJ. 2015. Locus coeruleus in time with the making of memories. Curr Opin Neurobiol 35: 87-94.

Sara SJ, Vankov A, Herve A. 1994. Locus coeruleus-evoked responses in behaving rats: a clue to the role of noradrenaline in memory. Brain Res Bull 35: 457-465.

Sara SJ, Roullet P, Przybyslawski J. 1999. Consolidation of memory for odor-reward association: beta-adrenergic receptor involvement in the late phase. Learn Mem 6: 88-96.
Segal M, Bloom FE. 1976. The action of norepinephrine in the rat hippocampus. IV. The effects of locus coeruleus stimulation on evoked hippocampal unit activity. Brain Res 107: 513-525.

Siapas AG, Wilson MA. 1998. Coordinated interactions between hippocampal ripples and cortical spindles during slow-wave sleep. Neuron 21: 1123-1128.

Singer AC, Carr MF, Karlsson MP, Frank LM. 2013. Hippocampal SWR activity predicts correct decisions during the initial learning of an alternation task. Neuron 77: 1163-1173.

Skaggs WE, McNaughton BL. 1996. Replay of neuronal firing sequences in rat hippocampus during sleep following spatial experience. Science 271: $1870-1873$.

Tronel S, Feenstra MG, Sara SJ. 2004. Noradrenergic action in prefrontal cortex in the late stage of memory consolidation. Learn Mem 11: 453-458.

Ul Haq R, Liotta A, Kovacs R, Rösler A, Jarosch MJ, Heinemann U, Behrens CJ. 2012. Adrenergic modulation of sharp wave-ripple activity in rat hippocampal slices. Hippocampus 22: 516-533.

Vandecasteele M, Varga V, Berényi A, Papp E, Barthó P, Venance L, Freund TF, Buzsáki G. 2014. Optogenetic activation of septal cholinergic neurons suppresses sharp wave ripples and enhances theta oscillations in the hippocampus. Proc Natl Acad Sci 111: 13535-13540.

Vertes RP, Kocsis B. 1997. Brainstem-diencephalo-septohippocampal systems controlling the theta rhythm of the hippocampus. Neuroscience 81: $893-926$.

Waterhouse BD, Moises HC, Woodward DJ. 1998. Phasic activation of the locus coeruleus enhances responses of primary sensory cortical neurons to peripheral receptive field stimulation. Brain Res 790: 33-44.

Wikenheiser AM, Redish AD. 2015. Decoding the cognitive map: ensemble hippocampal sequences and decision making. Curr Opin Neurobiol 32: 8-15.

Wilson MA, McNaughton BL. 1994. Reactivation of hippocampal ensemble memories during sleep. Science 265: 676-679.

Zhang W, Linden DJ. 2003. The other side of the engram: experience-driven changes in neuronal intrinsic excitability. Nat Rev 4: 885-900.

Received November 4, 2015; accepted in revised form February 28, 2016. 


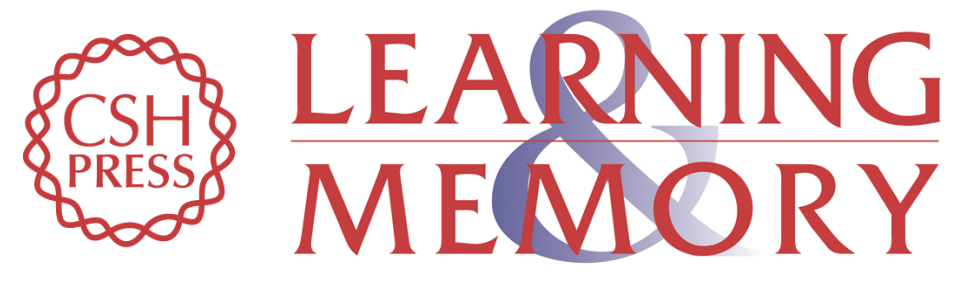

\section{Ripple-triggered stimulation of the locus coeruleus during post-learning sleep disrupts ripple/spindle coupling and impairs memory consolidation}

Yulia Novitskaya, Susan J. Sara, Nikos K. Logothetis, et al.

Learn. Mem. 2016, 23:

Access the most recent version at doi:10.1101/lm.040923.115

\section{Supplemental http://learnmem.cshlp.org/content/suppl/2016/04/11/23.5.238.DC1 \\ Material}

References This article cites 72 articles, 21 of which can be accessed free at: http://learnmem.cshlp.org/content/23/5/238.full.html\#ref-list-1

Creative This article is distributed exclusively by Cold Spring Harbor Laboratory Press for the Commons first 12 months after the full-issue publication date (see License http://learnmem.cshlp.org/site/misc/terms.xhtml). After 12 months, it is available under a Creative Commons License (Attribution-NonCommercial 4.0 International), as described at http://creativecommons.org/licenses/by-nc/4.0/.

Email Alerting Receive free email alerts when new articles cite this article - sign up in the box at the Service top right corner of the article or click here. 